\title{
Active and adaptive CFRP mirror using MFC piezoeletric actuator for thermal deformation and atmospheric aberration correction
}

Baghsiahi, Hadi, Jones, Martyn, Brooks, David, Doel, Peter

Hadi Baghsiahi, Martyn Jones, David Brooks, Peter Doel, "Active and adaptive CFRP mirror using MFC piezoeletric actuator for thermal deformation and atmospheric aberration correction," Proc. SPIE 11203, Advances in Optical Astronomical Instrumentation 2019, 112030P (3 January 2020); doi: 10.1117/12.2541341 


\title{
Active and Adaptive CFRP mirror using MFC Piezoeletric Actuator for Thermal Deformation and Atmospheric Aberration Correction
}

\author{
Hadi Baghsiahi*a, Martyn Jones ${ }^{\mathrm{b}}$, David Brooks ${ }^{\mathrm{a}}$, Peter Doel $^{\mathrm{a}}$ \\ ${ }^{a}$ Physics and Astronomy, University College London, UK; ${ }^{\mathrm{b}} \mathrm{Glyndwr}$ Innovation and composite \\ centre-Glyndwr University-UK
}

\begin{abstract}
Large precision composite mirrors for space missions and telescopes can be heavy, massive and expensive to fabricate. In this work, the mass of the mirrors is reduced by using space approved carbon fiber reinforced polymer (CFRP) material and employing micro-fiber composite (MFC) actuators for aberration correction and phase manipulation in an active/adaptive optical mirror system. The carbon fiber mirror is fabricated with 16 layers of prepreg composite MTM441/IMS65 carbon fiber and one layer of polishable resin. The layers are cured under pressure in an autoclave machine in a pressure and temperature-controlled environment. 2 different piezoelectric actuators, Push actuators and MFC actuators, are tested and the phase shift, form factor and surface deformation due to the active actuators are compared. Push actuators are the most common means of active optics. CFRP structure and surface deformation after actuator's effect are investigated theoretically using finite element analysis with the aid of COMSOL software and by optical experiments.
\end{abstract}

Keywords: CFRP, carbon fiber, CTE, active optics, adaptive optics, aberration correction, autoclave, cryogenic temperature, MFC, Piezoelectric actuator

\section{INTRODUCTION}

The opportunity to create a mirror substrate with a high specific stiffness is attractive to many industries and research sectors. Composite materials offer low mass and tailorable properties and are perfectly suited to a wide variety of applications. In this work, we attempt to reduce the mass of a mirror by using NASA space approved composite material for the reflector surface and micro fibre composite (MFC) actuators for Active optics to compensate for thermal surface deformation and atmosphere aberration correction. For a space mirror, toughness and stiffness are key issues and in active/adaptive optics applications, the applied force from the actuators might cause micro cracking or shape changing at the force point. Two different piezoelectric actuators are employed to create the active composite mirror; standard push actuators and MFC actuators.

\section{FABRICATION METHOD}

The replication from a mould is employed where a large mandrel, made from glass or metal, is formed with a convex/flat face that has the same form as the final mirror. The mandrel is polished to surface roughness of $5 \mu \mathrm{m}$ and figured to replicate the surface texture to the mirror. A liquid release agent is then deposed upon the mandrel. A layer of resin, either in liquid or partially cured film form, is applied to compensate for fibre print through. The number of plies, and their orientation is entirely dependent on how stiff the mirror is required.

The mandrel and the mirror substrate are then prepared for cure by creating a sealed bag using a high temperature polymer film. The substrate is then cured using an Autoclave, under vacuum, as per the recommended cycle advised by the manufacturer. Once cured at the mirror will separate easily from the mandrel and the surface of the mirror should have the replicated surface of the mandrel. The second process is to add a layer of a polishable resin on the surface to resolve the "fibre print through" problem and enable us to polish the surface to optical quality (Figure 1, Left). The mirror is then cut into a designed shape to remove the edges and the resin residue.

Advances in Optical Astronomical Instrumentation 2019, edited by Simon Ellis, Céline d'Orgeville, Proc. of SPIE Vol. 11203, 112030P · @ 2020 SPIE · CCC code: 0277-786X/20/\$21 · doi: 10.1117/12.2541341 

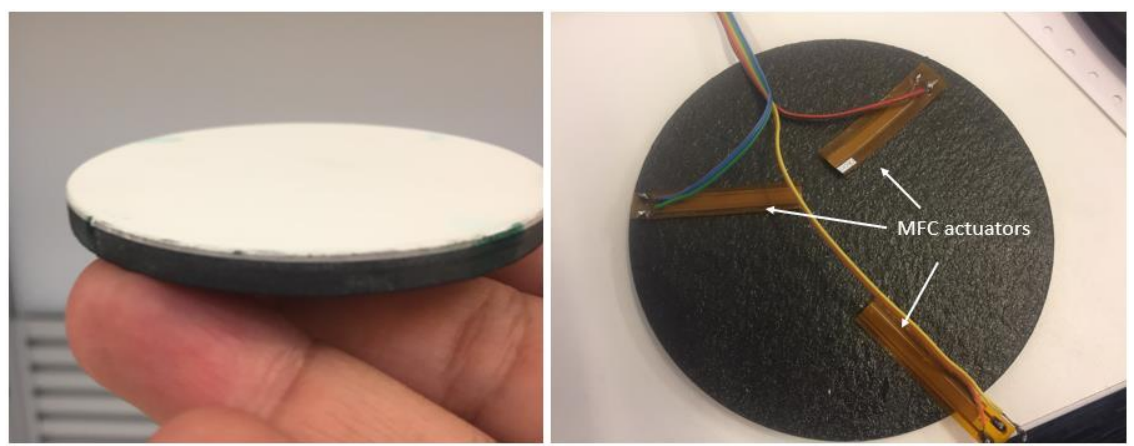

Figure 1: Left: A $70 \mathrm{~mm}$ diameter CFRP after applying resin on the top and cutting the edges. Right: Connecting MFC actuators to the substrate. Special glue is used to attach the MFCs to the back of the CFRP.

\section{ACTUATIONS FOR ADAPTIVE OPTICS}

Two different means of actuation we tested in this work. Push actuators are the most common Piezometric actuators used in adaptive optics. They deform the mirror surface based on the voltage which is applied to them. The force of the actuators are point effected and several actuators are required to achieve the required deformation and phase alteration (Figure 2)

MFC actuators are different with the push actuators in terms of required voltage and the way they apply force into the substrate. The MFCs are attached/glued into the back of the mirror and they are much lighter and require much less space (Figure 1, right). Also, they apply force into the mirror substrate as they expand, or contract depends of the voltage they are derived.
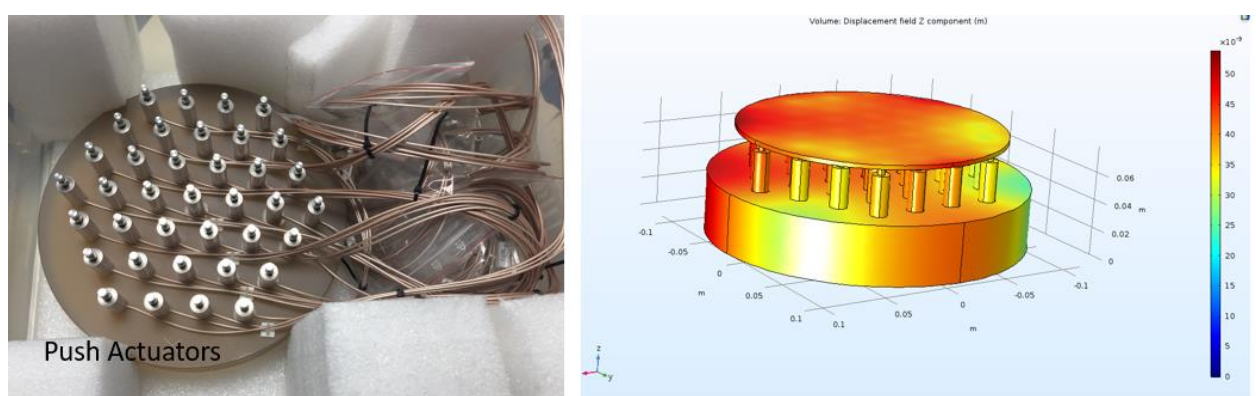

Figure 2: simulation results of using push actuators to deform the surface of a carbon fibre mirror to compensate for the atmospheric aberrations. Active/Adaptive optics prototype made in Optical science laboratory at UCL with a carbon fibre mirror.

Figure 2 and Figure 3 show some results for FEA analysis of the surface deformation due to the different types of actuators. We will present more results for different MFC structure designs and deformation created on the surface in the full paper. Also, experimental results of MFC actuators and push actuators will be compared to the COMSOL simulation results to drive the best design and method to be used in the full actuated active CFRP system. The advantages and dissadvantages of each method will be compared to find the most effective system for aberration correction and surface deformation of CFRPs. 


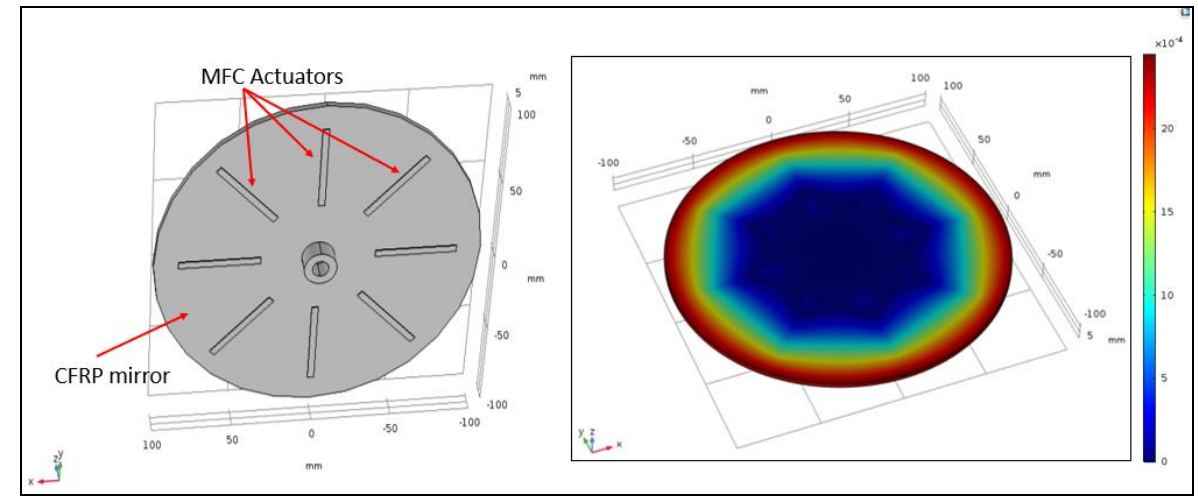

Figure 3: The simulation set up of CFRP and MFC attached to the back of the structure. Simulation results for using the MFC with 16 layers of CFRP

Proc. of SPIE Vol. 11203112030 P-3 